\title{
Extending the eNOE data set of large proteins by evaluation of NOEs with unresolved diagonals
}

\author{
Celestine N. Chi $^{1} \cdot$ Dean $\operatorname{Strotz}^{1} \cdot$ Roland Riek $^{1} \cdot$ Beat Vögeli $^{1}$
}

Received: 10 February 2015/ Accepted: 3 March 2015/Published online: 8 March 2015

(C) Springer Science+Business Media Dordrecht 2015

\begin{abstract}
The representation of a protein's spatial sampling at atomic resolution is fundamental for understanding its function. NMR has been established as the best-suited technique toward this goal for small proteins. However, the accessible information content rapidly deteriorates with increasing protein size. We have recently demonstrated that for small proteins distance restraints with an accuracy smaller than $0.1 \AA$ can be obtained by replacing traditional semi-quantitative Nuclear Overhauser Effects (NOEs) with exact NOEs (eNOE). The high quality of the data allowed us to calculate structural ensembles of the small model protein GB3 consisting of multiple rather than a single state. The analysis has been limited to small proteins because NOEs of spins with unresolved diagonal peaks cannot be used. Here we propose a simple approach to translate such NOEs into correct upper distance restraints, which opens access to larger biomolecules. We demonstrate that for $16 \mathrm{kDa}$ cyclophilin A the collection of such restraints extends the original 1254 eNOEs to 3471 .
\end{abstract}

Keywords Exact NOE - eNOE - NOESY - Structure calculation - Cyclophilin A

Roland Riek

roland.riek@phys.chem.ethz.ch

$\triangle$ Beat Vögeli

beat.voegeli@phys.chem.ethz.ch

1 Laboratory of Physical Chemistry, Vladimir-Prelog Weg 2, Swiss Federal Institute of Technology, ETH-Hönggerberg, 8093 Zurich, Switzerland

\section{Introduction}

The fundament of NMR-based structure determination of biomolecules is the Nuclear Overhauser Effect (NOE) between hydrogens (Wüthrich 1986; Neuhaus and Williamson 2000). Its traditional use however is only semiquantitative. We have recently demonstrated that for small proteins distance restraints with an accuracy smaller than $0.1 \AA$ can be obtained by careful analysis of a series of NOESY experiments (eNOE) (Kumar et al. 1981; Keepers and James 1984; Boelens et al. 1988, 1989; Vögeli et al. 2009, 2010; Vögeli 2014). Subsequently, we used these exact restraints to calculate structural ensembles of the small model protein GB3 consisting of multiple rather than a single state (Vögeli et al. 2012, 2013; Vögeli 2014). Such ensembles were in significantly better agreement with the eNOEs and thus represent a more realistic sampling of the conformational space than single-state calculations (Brüschweiler et al. 1991, 1992; Bonvin et al. 1994; Lindorff-Larsen et al. 2005; Olsson et al. 2014; Mantsyzov et al. 2014). These representations open an avenue for a comprehensive description of a protein's structural landscape and dynamics at atomic resolution (Fenwick et al. 2011; Ravera et al. 2014; Vögeli et al. 2014; Torchia 2015). In order to extend the eNOE-based ensemble structure determination to larger biomolecules, we propose here a simplified approach to translate an NOE into a correct upper distance restraint and demonstrate it for the $16 \mathrm{kDa}$ cis/trans isomerase human cyclophilin A (Handschumacher et al. 1984; Clubb et al. 1994; Ottiger et al. 1997; Wang and Heitman 2005), for which 3471 such restraints were collected.

In the eNOE analysis, the NOESY cross-peak intensities have to be normalized to back-predicted intensities of the diagonal peaks for zero mixing time (Kumar et al. 1981; 
Keepers and James 1984; Boelens et al. 1988, 1989; Vögeli et al. 2009, 2010; Vögeli 2014). As a consequence, cross peaks arising from spin pairs with overlapped diagonal peaks cannot be converted into exact distance restraints, which reduces the number of eNOE-derived distance restraints significantly. Supplementing eNOE with conventional NOE restraints would adversely impact on the multiple-state ensembles as the restraints are calibrated in an overall statistical manner. A comparison of upper distance limits either obtained from conventional NOEs following the standard CYANA (Güntert 2009) procedure or from our eNOE protocol is shown in Fig. 1. Counting bidirectional NOEs once, $10 \%$ of the individual restraints potentially violate the true distances and would enforce false separation of states in multi-state ensembles. On the other hand, $55 \%$ of the restraints are at least $1 \AA$ less restrictive than those obtained from eNOEs, which results in a large loss of information.

Omission of the conventional NOEs typically reduces the overall NOE data set by hundreds of restraints and the relative loss increases with increasing protein size. This effect is most pronounced for large protein systems because the chemical shift overlap increases approximately exponentially with increasing protein size (Mumenthaler et al. 1997; Güntert 2003). For example, while for the 56-residue protein GB3 823 eNOE- and 1041 NOE-derived distance restraints were collected (Vögeli et al. 2012, 2013), the respective numbers are 1254 and 3471 for the 165-residue protein cyclophilin. In the following, we

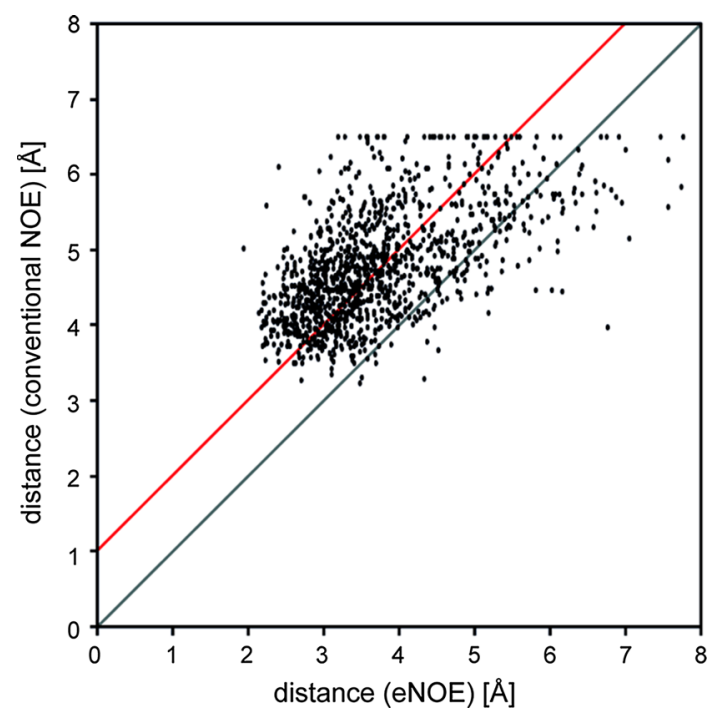

Fig. 1 Comparison of upper distance limits obained from conventional NOEs with CYANA (Güntert 2009) and limits derived from eNOEs for cyclophilin A. Data points below the black line denote limits that potentially violate the true distances. Data points above the red line denote limits that are at least $1 \AA$ less restrictive than those obtained from eNOEs introduce a protocol to rescue the conventional NOEs and translate them into quantitative upper distance restraints. We show that restraints from cross peaks with overlapped diagonal peaks can provide a relatively tight upper limit, while never being lower than the true value. In such a way, multiple-state ensembles can be significantly better defined without causing artificial state separation.

\section{Results and discussion}

We find that the intensities of non-overlapped diagonal peaks in a series of 3D $\left[{ }^{15} \mathrm{~N},{ }^{13} \mathrm{C}\right]$-resolved $\left[{ }^{1} \mathrm{H},{ }^{1} \mathrm{H}\right]$-NOESY spectra of human cyclophilin back-calculated to zero mixing time are dispersed over more than one order of

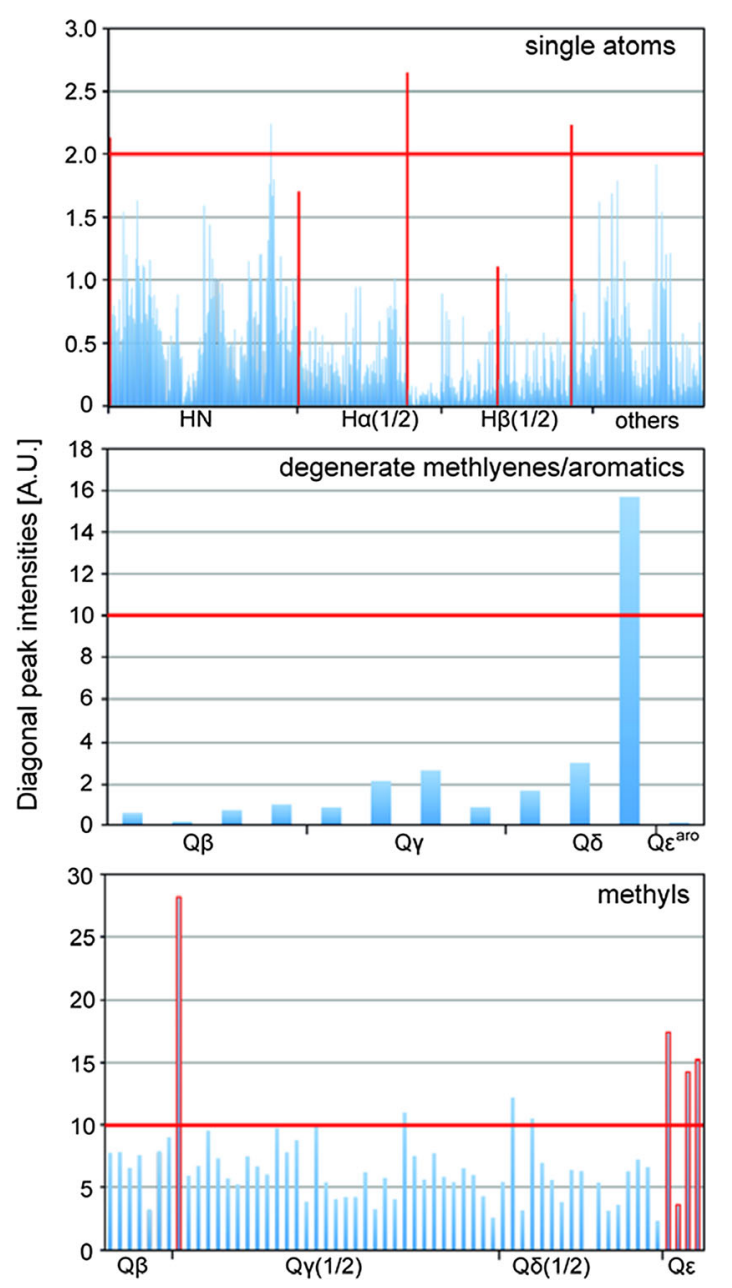

Fig. 2 Back-calculated intensities of diagonal peaks in a 3D $\left[{ }^{15} \mathrm{~N},{ }^{13} \mathrm{C}\right]$-resolved $\left[{ }^{1} \mathrm{H},{ }^{1} \mathrm{H}\right]$-NOESY spectrum of human cyclophilin $\mathrm{A}$ at zero mixing time. Intensities of single ${ }^{1} \mathrm{H}$, degenerate methylene or aromatic, and methyl spins are shown in the top, middle, and bottom panel, respectively. The red horizontal lines indicate the chosen intensities used for the calculation of the upper distance limits from generic normalized eNOEs. Intensities of spins located in the termini and $\mathrm{Q}^{\varepsilon}$ methyls of methionine are framed in red 
magnitude (Fig. 2). Non-uniform relaxation properties, scalar couplings, incomplete equilibration of the intrinsic pre-scan polarization (although an interscan delay of $1 \mathrm{~s}$ was chosen here), and suboptimal performance of the pulses on the hetero-nuclei due to offset effects cause differentiation of the intensities. Interestingly, all outliers with large intensities are located to the highly mobile termini and to the outmost positions of long side chains. Most of them are atoms in the N-terminal residues 1 and 2, the C-terminal residue 165 and the $\mathrm{Q}^{\varepsilon}$ methyl groups of methionine (colored red in Fig. 2).

Given the simple identification rule for maximum outliers, it is straightforward to set an upper practical limit to the expected intensities even if the diagonal peaks cannot be analyzed due to peak overlap. The horizontal red lines in Fig. 2 indicate such limits that are chosen larger than almost any intensity not corresponding to the identified risk group (i.e. terminal residues 1, 2, and 165, and the four methionine methyl groups $\mathrm{Q}^{\varepsilon}$ of residues $61,100,136$, and 142). It is our rational that if a cross-peak buildup can be fitted, the curve may be normalized to the corresponding generic diagonal intensity resulting in a lower limit for the cross-relaxation rate. The cross-relaxation rate, in turn, can then be converted into an upper distance limit using the rotational correlation time measured from ${ }^{15} \mathrm{~N}$ relaxation data. This upper distance limit is still of high quality since an offset in the diagonal intensity is greatly reduced in the distance due to the $1 / \mathrm{r}^{6}$ dependency of the NOE. For example, if the generic diagonal intensity is twice the true value, the upper distance limit is only $12 \%$ larger than the true value. In the following, we refer to such upper limits as distances derived from generic normalized eNOEs.

In principle, it is also possible to define a generic lower limit for the diagonal intensities. The use of such a limit would result in lower distance limits from cross peaks without available diagonal peak intensities. As opposed to upper intensity limits, lower limits may easily be violated in specific cases due to intensity reduction via exchange broadening or suboptimal pulses. Therefore, it is not recommended to use lower distance limits from generic normalized eNOEs.

To test the validity of the approach, we compare in the following upper distance limits extracted from generic normalized eNOEs to those from regular eNOEs of human cyclophilin A, respectively. 1254 upper limit restraints were obtained from ${ }^{15} \mathrm{~N},{ }^{13} \mathrm{C}$-resolved $\left[{ }^{1} \mathrm{H},{ }^{1} \mathrm{H}\right]$-NOESY experiments with mixing times $15,20,25,30,40$ and $50 \mathrm{~ms}$. Both types of experimental eNOEs were corrected for spin diffusion using the eNORA program (Orts et al. 2012). The correlations between the resulting upper distance limits are plotted in Fig. 3. The 44 comparisons of the risk group are colored red. Excluding those, among the remaining 1210 distances there are no violations larger

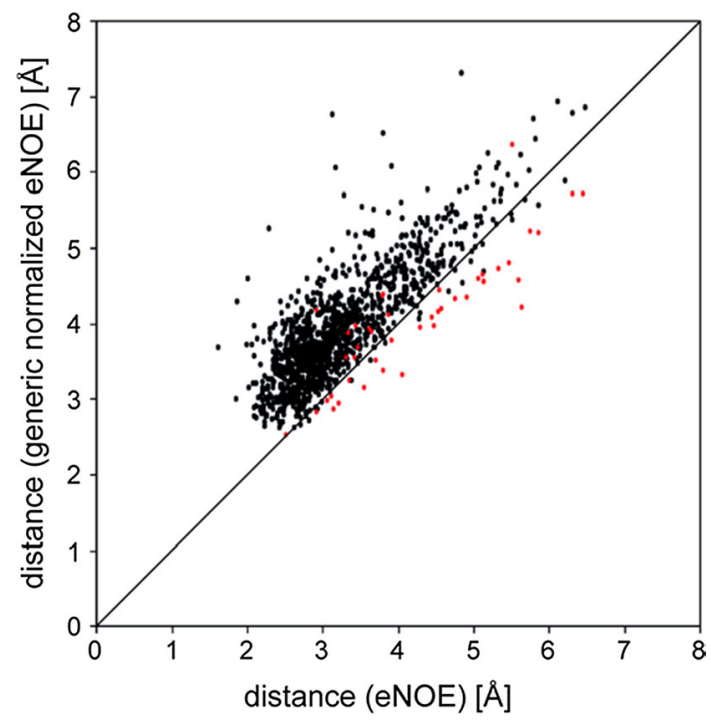

Fig. 3 Upper distance limits obtained from generic normalized eNOEs versus those from eNOEs. If the cross-relaxation rate is normalized to the diagonal peak intensity of a terminal residue or $\mathrm{Q}^{8}$ of methionine, it is marked red. The black line shows slope 1

than $0.5 \AA$, two with violations larger than $0.3 \AA$, and three more restraints are violated by more than $0.2 \AA$. The largest one is caused by the $\mathrm{Q}^{\gamma 2}(\mathrm{Val} 20)-\mathrm{H}^{\mathrm{N}}(\mathrm{Ser} 21)$ spin pair with $0.43 \AA$. Inspection reveals that it is caused by the fact that only one cross peak could be used for the eNOE, while both were used for the generic normalized eNOE. Indeed, comparison of the cross-relaxation rate corresponding to the same cross peak shows a nearly identical value. To be cautious, these upper limit restraints are multiplied by a factor of 1.2 for the structure calculation, as done for the unidirectional eNOE-derived distances (Vögeli et al. 2012, 2013). Thus, no restraints are violated and the presented approach is highly reliable. It is worth to mention that it is possible that the risk group for another system may also include other highly flexible segments such as a protein loop. Such segments should be easy to identify by resolved diagonal peaks originating from protons in the segment. Although not the case in our study (methione being the exception), other candidates are ends of long side chains such as those of lysines. These candidates are expected to cause violations in the structure determination process and thus also to be identified.

Importantly, 2217 additional generic normalized eNOEs were determined without an eNOE counterpart enlarging the number of restraints for cyclophilin A by a factor of almost 2. To show their impact, we calculated structures with the software package CYANA (Güntert 2009) using either only the 1254 upper and lower distance limits from eNOEs or supplemented with the limits from the generic normalized eNOEs (omitting the ones from the risk group, Tables 1 and 2). Although the 1254 restraints from the 
Table 1 Impact of generic normalized eNOEs on CYANA structures of cyclophilin A: statistics of the ordered residues

\begin{tabular}{|c|c|c|c|}
\hline \# Upper/lower limits from eNOEs & $\begin{array}{l}\text { \# Upper limits from } \\
\text { generic normalized eNOEs }\end{array}$ & $\begin{array}{l}\text { R.m.s.d. to the mean }{ }^{\mathrm{a}} \text {, } \\
\text { backbone }(\AA)\end{array}$ & $\begin{array}{l}\text { R.m.s.d. to the mean }{ }^{\text {a }} \text {, } \\
\text { all heavy atoms }(\AA)\end{array}$ \\
\hline \multicolumn{4}{|l|}{20 single-state structures } \\
\hline $1254 / 1254$ & 0 & $0.84 \pm 0.11$ & $1.27 \pm 0.13$ \\
\hline $1254 / 1254$ & 2144 & $0.59 \pm 0.07$ & $0.97 \pm 0.07$ \\
\hline $854 / 854$ & 0 & $1.23 \pm 0.17$ & $1.69 \pm 0.16$ \\
\hline $854 / 854$ & 2144 & $0.65 \pm 0.12$ & $1.00 \pm 0.11$ \\
\hline $454 / 454$ & 0 & $1.97 \pm 0.22$ & $2.63 \pm 0.19$ \\
\hline $454 / 454$ & 2144 & $0.72 \pm 0.11$ & $1.11 \pm 0.09$ \\
\hline 4537 conventional NOEs & & $0.90 \pm 0.11$ & $1.30 \pm 0.12$ \\
\hline \multicolumn{4}{|l|}{20 two-states ensembles } \\
\hline $1254 / 1254$ & 0 & $1.19 \pm 0.14$ & $1.71 \pm 0.17$ \\
\hline $1254 / 1254$ & 2144 & $0.97 \pm 0.12$ & $1.56 \pm 0.20$ \\
\hline 4537 conventional NOEs & & $1.23 \pm 0.14$ & $1.79 \pm 0.16$ \\
\hline
\end{tabular}

${ }^{a}$ Residues 3-65, 77-163

Table 2 Impact of generic normalized eNOEs on CYANA structures of cyclophilin A: statistics of all residues

\begin{tabular}{|c|c|c|c|c|}
\hline $\begin{array}{l}\text { \# Upper/lower limits } \\
\text { from eNOEs }\end{array}$ & $\begin{array}{l}\text { \# Upper limits from generic } \\
\text { normalized eNOEs }\end{array}$ & $\begin{array}{l}\text { R.m.s.d. to the mean }{ }^{\mathrm{a}} \text {, } \\
\text { backbone }(\AA)\end{array}$ & $\begin{array}{l}\text { R.m.s.d. to the mean }{ }^{\mathrm{a}} \text {, all } \\
\text { heavy atoms }(\AA)\end{array}$ & $\begin{array}{l}\text { eNOE }+ \text { generic normalized } \\
\text { eNOE violations }\end{array}$ \\
\hline \multicolumn{5}{|c|}{20 single-state structures } \\
\hline $1254 / 1254$ & 0 & $1.21 \pm 0.21$ & $1.66 \pm 0.21$ & 57 \\
\hline $1254 / 1254$ & 2144 & $1.23 \pm 0.18$ & $1.52 \pm 0.15$ & 153 \\
\hline $854 / 854$ & 0 & $1.52 \pm 0.22$ & $2.00 \pm 0.21$ & 24 \\
\hline $854 / 854$ & 2144 & $1.32 \pm 0.31$ & $1.62 \pm 0.27$ & 76 \\
\hline $454 / 454$ & 0 & $2.40 \pm 0.33$ & $3.02 \pm 0.28$ & 7 \\
\hline $454 / 454$ & 2144 & $1.27 \pm 0.22$ & $1.58 \pm 0.16$ & 29 \\
\hline \multicolumn{2}{|c|}{4537 conventional NOEs } & $1.39 \pm 0.34$ & $1.79 \pm 0.30$ & 15 \\
\hline \multicolumn{5}{|c|}{20 two-states ensembles } \\
\hline $1254 / 1254$ & 0 & $1.57 \pm 0.26$ & $2.11 \pm 0.25$ & 10 \\
\hline $1254 / 1254$ & 2144 & $1.32 \pm 0.16$ & $1.89 \pm 0.21$ & 12 \\
\hline \multicolumn{2}{|c|}{4537 conventional NOEs } & $1.60 \pm 0.27$ & $2.16 \pm 0.25$ & 0 \\
\hline
\end{tabular}

${ }^{a}$ Residues 2-164

eNOEs alone define the structure of cyclophilin A relatively well, the r.m.s. deviation from the mean structure in the ordered segments is reduced by $0.25 \AA$ for the backbone, and by $0.30 \AA$ for all heavy atoms, when the complete data set is used (Fig. 4). A structure calculation with 4537 upper distance limits from conventional NOEs produces r.m.s.d. values that are 0.06 and $0.03 \AA$ larger than those obtained from regular eNOEs only, and 0.31 and $0.33 \AA$ larger than those derived from the complete eNOE data set. These effects are largely masked by the high flexibility of the termini and the loop comprising residues 66-76 if the statistics are taken over the entire molecule (Table 2).
An increased fraction of the generic normalized eNOEs is expected for larger systems due to more resonance overlap and as also verified by ongoing work in our laboratory. We simulated such a scenario for cyclophilin by random deletion of 400 or 800 upper and lower distance limits from the original eNOE data set. The impact of the generic normalized eNOEs becomes very pronounced as demonstrated in Fig. 4 and Tables 1 and 2. When using a $5: 2$ (2144:854) instead of the original 5:3 (2144:1254) ratio of the counts of generic normalized eNOEs to regular eNOEs, the structural r.m.s.d. in the ordered segments of cyclophilin comprising residues $3-65$ and $77-163$ is 
Fig. 4 Impact of supplement of the generic normalized eNOEs on the structure calculation of cyclophilin A. Structures calculated using 1254 eNOEs supplemented with 2217 generic normalized eNOEs (left), 1254 eNOE alone (middle) and 4537 conventional NOEs (right)
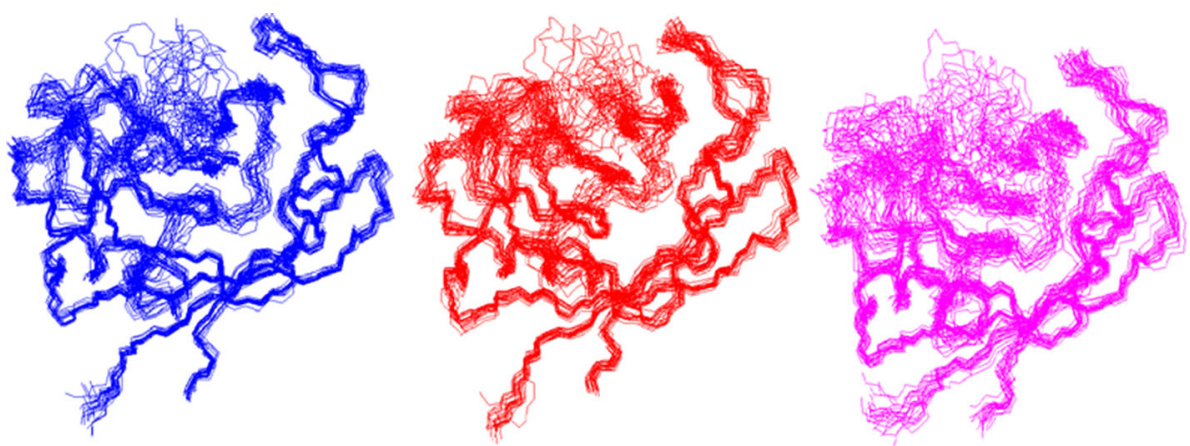

reduced by ca. $0.6 \AA$ for both the backbone as well as all the heavy atoms upon exclusion of the generic normalized eNOEs (Table 1). The r.m.s.d. reduction is more than $1.2 \AA$ (backbone) and $1.4 \AA$ (all heavy atoms) for a 5:1 (2144:454) ratio. Although the r.m.s.d. reductions are weaker for the entire molecule (Table 2), similar trends as those for the ordered segments are observed.

While the lower r.m.s. deviations for the eNOE data provide a measure of the increased completeness over the conventional NOE networks, it does not quantify the quality of the data. We observe similar trends for the r.m.s. deviations in two-states ensembles (Table 1). However, we observe that bundles derived from conventional NOEs do not provide clear state separation, while only the ensemble calculated from eNOEs and generic normalized eNOEs produce consistently two states even in the loops (to be published elsewhere).

In conclusion, we introduced a protocol to enlarge the eNOE data set substantially without adding semi-quantitative restraints such as those derived from conventional NOEs that may contradict true distances. Because this data does not enforce false separation of states, eNOE-based multiple-state ensemble structure calculation becomes applicable to larger, biologically relevant proteins.

\section{Materials and methods}

\section{Sample preparation}

The gene enconding the full-length human cyclophilin was sub-cloned in a pRSET/A vector (Invitrogen) containing an N-terminal His-tag with a thrombin cleavage site (MHHHHHHLVPRGS). Expression and purification will be described elsewhere (Chi et al. manuscript submitted). Briefly, the cDNA containing the cyclophilin gene was transformed into E. coli BL21 plyS cells and plated on an ampicillin/chloramphenicol-containing plate. A 11 culture in M9 medium for ${ }^{15} \mathrm{~N} /{ }^{13} \mathrm{C}$ - or ${ }^{15} \mathrm{~N}$-labeling was initiated and cells were grown to an $\mathrm{OD}_{600}$ of 0.9. Protein expression was initiated by adding $1 \mathrm{mM}$ IPTG (isopropyl $\beta$-D-thiogalactopyranoside). Cells were then allowed to express over night at $18{ }^{\circ} \mathrm{C}$. Cells were harvested, re-suspended in purification buffer $(10 \mathrm{mM}$ Tris/ $\mathrm{HCl})$ and lysed by French press and spun at 40,000 $\mathrm{g}$ for $20 \mathrm{~min}$. The supernatant was filtered and loaded onto a nickel (II)-charged chelating Sepharose FF column (Amersham Biosciences), equilibrated with purification buffer as above and washed with $400 \mathrm{ml}$ of the same buffer. The bound protein was eluted with $250 \mathrm{mM}$ imidazole at $\mathrm{pH} 7.9$, in aliquots of $12 \mathrm{ml}$. Fractions containing partially pure proteins were pooled, dialyzed for $2 \mathrm{~h}$ and passed through a DEAE S-column equilibrated with purification buffer. The His-tag was cleaved off by incubating in thrombin for $3 \mathrm{~h}$ at room temperature. Pure cyclophilin was collected as flow-through by passing the digested fractions through a nickel (II)-charged chelating Sepharose FF column equilibrated with purification buffer. The purity was checked on SDS PAGE stained with coomassie brilliant blue and its identity confirmed by Matrix Assisted Laser Desorption Ionization time-of-flight mass spectrometry (MALDI-TOF). The final NMR samples contained protein at a concentrations of $1-2 \mathrm{mM}$ (as determined by absorption measurements) in $10 \mathrm{mM}$ sodium phosphate, pH 6.5, $5 \mathrm{mM}$ DTT, $0.01 \% \mathrm{NaNO}_{3}$ and $3 \% \mathrm{D}_{2} \mathrm{O}$.

\section{NMR spectroscopy and data analysis}

All NMR experiments were run on Bruker 600 and $700 \mathrm{MHz}$ spectrometers equipped with triple resonance cryogenic probes at $299 \mathrm{~K}$. Data were processed and analysed with NMRpipe (Delaglio et al. 1995) and CCPnmr (Vranken et al. 2005).

For assignment purposes 3D HNCACB, ${ }^{15} \mathrm{~N}$-resolved $\left[{ }^{1} \mathrm{H},{ }^{1} \mathrm{H}\right]$-NOESY-HSQC, $\quad{ }^{15} \mathrm{~N}$-resolved HMQC- $\left[{ }^{1} \mathrm{H},{ }^{1} \mathrm{H}\right]-$ NOESY, ${ }^{15} \mathrm{~N}$-resolved $\left[{ }^{1} \mathrm{H},{ }^{1} \mathrm{H}\right]$-TOCSY-HSQC and ${ }^{13} \mathrm{C}$ resolved HCCH-TOCSY spectra were recorded (Cavanagh et al. 2007). Cross peak buildups and diagonal peak decays were probed with $3 \mathrm{D}{ }^{15} \mathrm{~N} /{ }^{13} \mathrm{C}$-resolved $\left[{ }^{1} \mathrm{H},{ }^{1} \mathrm{H}\right]$-NOESYHMQC experiments using mixing times of 15, 20, 25, 30, 40 and $50 \mathrm{~ms}$ (Vögeli et al. 2013). 
1254 upper and lower distance restraints from eNOEs were determined with the eNORA software package (Orts et al. 2012) following the previously established protocol: (1) The diagonal intensity of each residue was fitted monoexponentially to back-predict the intensity at zero mixing time and to obtain the autorelaxation rate. (2) The initial intensity was then used to normalize the cross peak intensity measured at the various mixing times. (3) These normalized values were fitted to determine the cross-relaxation rate. (4) A correction for spin diffusion effects was estimated from simulations of apparent cross-relaxation rates from an input structure [lowest energy conformer from pdb code 1oca (Ottiger et al. 1997)] using the full relaxation matrix method (Orts et al. 2012). (5) The upper and lower distance restraints were then set following the established protocol (Vögeli et al. 2013). Magnetically equivalent protons were treated by $r^{-6}$-summation rather than a pseudo-atom approach as used previously (Vögeli et al. 2013).

3471 generic normalized eNOEs were generated with the same eNORA protocol as used for the eNOEs with the following exceptions: (1) the back-predicted diagonal peak intensities at zero mixing time were plotted as shown in Fig. 2. Separate generic diagonal peak intensities were chosen for three different classes of peaks, the first including all single atoms, the second the degenerate and aromatic atoms, and the third the methyl groups, respectively. The values were set to 2, 10 and 10 (note, the scale for peak intensities comes in arbitrary units). The values were selected such that they are larger than all diagonal intensities except for a few outliers that are easily categorized as the risk group (see main text). The resulting upper distance limits are rather insensitive to the particular choice due to the $r^{-6}$ dependency of the cross-relaxation rate.

4537 conventional NOEs were extracted from the 3D ${ }^{15} \mathrm{~N} /{ }^{13} \mathrm{C}$-resolved $\left[{ }^{1} \mathrm{H},{ }^{1} \mathrm{H}\right]$-NOESY-HSQC experiment with a mixing time of $50 \mathrm{~ms}$. The NOE intensities $I$ were converted into upper distance limits $r^{\text {CYANA }}$ using the standard CYANA procedure (Güntert 2009):

$r^{\text {CYANA }}=\left[r^{\text {reference }} * \text { median }(\text { Iof all cross peaks }) / I\right]^{1 / 6}$

For $r^{\text {reference } 6}$, we chose the default setting of $4.0 \AA$. Distances larger than $6.5 \AA$ were set to $6.5 \AA$.

${ }^{15} \mathrm{~N} R_{1}, R_{1}$, and $R_{2}$ relaxation rates were recorded with standard $\left[{ }^{15} \mathrm{~N},{ }^{1} \mathrm{H}\right]$-TROSY type experiments (Cavanagh et al. 2007) and resulted in a overall tumbling time $\tau_{\mathrm{c}}$ of $9.2 \mathrm{~ns}$.

\section{Structure calculation}

Upper and lower distance restraints from eNOEs and generic normalized eNOEs or from conventional NOEs were used together with conservative $\phi$ and $\psi$ dihedral angle restraints from $\mathrm{C}^{\alpha}$ chemical shifts for structure determination. The weight oft the dihedral angle restraints were reduced to zero in the final part of the calculation. Structural coordinates were determined with version 3.97 of the program CYANA (Güntert 2009). Calculations were done with 200,000 torsion angle dynamics steps for 200 conformers with random torsion angles by simulated annealing. The 20 conformers with the lowest final target function values were selected and analyzed. The coordinates of the single state bundle calculated from the complete data set consisting of the eNOEs and the generic normalized eNOEs together with the upper/lower distance limit tables are deposited in the PDB/BMRB (pdb code 2MZU; bmrb accession code 25502). The two structural states were calculated simultaneously and averaged for the two-state ensemble. A weak harmonic well potential with bottom width of $1.2 \AA$ was used to keep identical heavy atoms from the different states together following the previous proposal (Vögeli et al. 2012, 2013).

Acknowledgments This work was supported by the Swedish Research council and a Wenner-Gren post doc fellowship to C.N C, and the Swiss National Science Foundation with Grant 140214 and by ETH Research Grant ETH-04 13-1 to B.V.

\section{References}

Boelens R, Koning TMG, Kaptein R (1988) Determination of biomolecular structures from proton-proton NOEs using a relaxation matrix approach. J Mol Struct 173:299-311

Boelens R, Koning TMG, Van der Marel GA, Van Boom JH, Kaptein $R$ (1989) Iterative procedure for structure determination from proton-proton NOEs using a full relaxation matrix approach Application to a DNA octamer. J Magn Reson 82:290-308

Bonvin AMJJ, Boelens R, Kaptein R (1994) Time- and ensembleaveraged direct NOE restraints. J Biomol NMR 4:143-149

Brüschweiler R, Blackledge M, Ernst RR (1991) Multi-conformational peptide dynamics derived from NMR data: a new search algorithm and its application to antamanide. J Biomol NMR $1: 3-11$

Brüschweiler R, Roux B, Blackledge M, Griesinger C, Karplus M, Ernst RR (1992) Influence of rapid intramolecular motions on NMR cross-relaxation rates. A molecular dynamics study of antamanide in solution. J Am Chem Soc 114:2289-2302

Cavanagh J, Fairbrother WJ, Palmer AG, Rance M, Skleton NJ (2007) Protein NMR spectroscopy. Principles and practice. Academic Press, San Diego

Clubb RT, Ferguson SB, Walsh CT, Wagner G (1994) Threedimensional solution structure of Escherichia coli periplasmic cyclophilin. Biochemistry 33:2761-2772

Delaglio F, Grzesiek S, Vuister GW, Zhu G, Pfeifer J, Bax A (1995) NMRPipe: a multidimensional spectral processing system based on UNIX pipe. J Biomol NMR 6:277-293

Fenwick RB, Esteban-Martin S, Salvatella X (2011) Understanding biomolecular motion, recognition, and allostery by use of conformational ensembles. Eur Biophys J 40:1339-1355

Güntert P (2003) Methods and principles in medicinal chemistry, vol 16. Wiley-VCH, Weinheim, pp 39-66 
Güntert P (2009) Automated structure determination from NMR spectra. Eur Biophys J 38:129-143

Handschumacher E, Harding MW, Rice J, Drugge RJ, Speicher DW (1984) Cyclophilin: a specific cytosolic binding protein for cyclosporin A. Science 226:544-547

Keepers JW, James TL (1984) A theoretical study of distance determinations from NMR. Two-dimensional nuclear Overhauser effect spectra. J Magn Reson 57:404-426

Kumar A, Wagner G, Ernst RR, Wüthrich K (1981) Buildup rates of the nuclear Overhauser effect measured by two-dimensional proton magnetic resonance spectroscopy: implication for studies of protein conformation. J Am Chem Soc 103:3654-3658

Lindorff-Larsen K, Best RB, DePristo MA, Dobson CM, Vendruscolo $M$ (2005) Simultaneous determination of protein structure and dynamics. Nature 433:128-132

Mantsyzov AB, Maltsev AS, Ying J, Shen Y, Hummer G, Bax A (2014) A maximum entropy approach to the study of residuespecific backbone angle distributions in a-synuclein, an intrinsically disordered protein. Prot Sci 23:1275-1290

Mumenthaler C, Güntert P, Braun W, Wüthrich K (1997) Automated combined assignment of NOESY spectra and three-dimensional protein structure determination. J Biomol NMR 10:351-362

Neuhaus D, Williamson MP (2000) The nuclear Overhauser effect in structural and conformational analysis. Wiley, New York

Olsson S, Vögeli B, Cavalli A, Boomsma W, Ferkinghoff-Borg J, Lindorff-Larsen K, Hamelryck T (2014) Probabilistic determination of native state ensembles of proteins. J Chem Theory Comput 10:3484-3491

Orts J, Vögeli B, Riek R (2012) Relaxation matrix analysis of spin diffusion for the NMR structure calculation with eNOEs. J Chem Theory Comput 8:3483-3492

Ottiger M, Zerbe O, Güntert P, Wüthrich K (1997) The NMR solution conformation of unligated human cyclophilin A. J Mol Biol 272:64-81
Ravera E, Salmon L, Fragai M, Parigi G, Al-Hashimi H, Luchinat C (2014) Insights into domain-domain motions in proteins and RNA from solution NMR. Acc Chem Res 47:3118-3126

Torchia DA (2015) NMR studies of dynamic biomolecular conformational ensembles. Prog Nucl Magn Reson Spectrosc 84-85:14-32

Vögeli B (2014) The nuclear Overhauser effect from a quantitative perspective. Prog Nucl Magn Reson Spectrosc 78:1-46

Vögeli B, Segawa T, Leitz D, Sobol A, Choutko A, Trzesniak D, Van Gunsteren W, Riek R (2009) Exact distances and internal dynamics of perdeuterated ubiquitin from NOE buildups. J Am Chem Soc 131:17215-17225

Vögeli B, Friedmann M, Leitz D, Sobol A, Riek R (2010) Quantitative determination of NOE rates in perdeuterated and protonated proteins: practical and theoretical aspects. J Magn Reson 204:290-302

Vögeli B, Kazemi S, Güntert P, Riek R (2012) Spatial elucidation of motion in proteins by ensemble-based structure calculation using exact NOEs. Nat Struct Mol Biol 19:1053-1057

Vögeli B, Güntert P, Riek R (2013) Multiple-state ensemble structure determination from eNOE spectroscopy. Mol Phys 111:437-454

Vögeli B, Orts J, Strotz D, Chi C, Minges M, Wälti MA, Güntert P, Riek R (2014) Towards a true protein movie: a perspective on the potential impact of the ensemble-based structure determination using exact NOEs. J Magn Reson 241:53-59

Vranken WF, Boucher W, Stevens TJ, Fogh RH, Pajon A, Llinas M, Ulrich EL, Markley JL, Ionides J, Laue ED (2005) The CCPN data model for NMR spectroscopy: development of a software pipeline. Proteins 59:687-696

Wang P, Heitman J (2005) The cyclophilins. Genome Biol 6:226

Wüthrich K (1986) NMR of proteins and nucleic acids. Wiley, New York 